\title{
Characterizing Frequency Stability Measurements having Multiple Data Gaps
}

This paper was downloaded from TechRxiv (https://www.techrxiv.org).

\section{LICENSE}

CC BY 4.0

SUBMISSION DATE / POSTED DATE

$15-10-2021 / 20-10-2021$

\section{CITATION}

Howe, David; Schlossberger, Noah (2021): Characterizing Frequency Stability Measurements having Multiple Data Gaps. TechRxiv. Preprint. https://doi.org/10.36227/techrxiv.16821355.v1

$\mathrm{DOI}$

10.36227/techrxiv.16821355.v1 


\title{
Characterizing Frequency Stability Measurements having Multiple Data Gaps
}

\author{
D. A. Howe, Fellow, IEEE, and N. Schlossberger
}

\begin{abstract}
Time series measurements with data gaps (dead times) prevent accurate computations of frequency stability variances such as the Allan variance (AVAR) and its square-root the Allan deviation (ADEV). To extract frequency distributions, time series data must be sequentially ordered and equally spaced. Data gaps, particularly large ones, make ADEV estimates unreliable. Gap imputation by interpolation, zero-padding, or adjoining live segments, all fail in various ways. We have devised an algorithm that fills gaps by imputing an extension of preceding live data and explain its advantages. To demonstrate the effectiveness of the algorithm, we have implemented it on 512length original datasets and have removed $30 \%$ (150 values). The resulting data is consistent with the original in all three major criteria: the noise characteristic, the distribution, and the ADEV levels and slopes. Of special importance is that all ADEV measurements on the imputed data set lie within $90 \%$ confidence of the statistic for the original dataset.
\end{abstract}

Index Terms - ADEV, AVAR, clock, deviation, frequency, modified, noise, oscillator, power-law, stability, standard, time, variance.

\section{INTRODUCTION}

Time series measurements of clocks and oscillators must be equally spaced to characterize noise models using ADEV. Random gaps ("dead times") cause ADEV and related statistics to mischaracterize noise or to fail outright for gaps greater than $10 \%$ of the data set. Trying to characterize noise with gaps introduces biases and significant ADEV uncertainty. Sec. II reviews frequency-time statistics and shows how data extensions by reflection instead of by common periodic extensions are independent. Sec. III gives the imputation algorithm. Sec. IV shows simulation results of ADEV on data before a $30 \%$ segment is removed and after the imputation algorithm is applied to the gapped data.

\section{FREQUENCY STABILITY}

Frequency stability is characterized by the Allan deviation, designated as $\sigma_{\mathrm{y}}(\tau)$, ADEV or sq-rt AVAR and its related statistical estimators include the modified-Allan deviation (MDEV), THEO, TDEV, TOTDEV, etc. These estimators are in the class of "frequency-time" statistics that distinguish between different noise types [1-3]. Discrete-continuous functions of either time error $x(t)$ or fractional-frequency error $y(t)$ produce Power Spectral Density (PSD) functions $S_{x}(f)$ and $S_{y}(f)$, respectively, by use of FFT and DFT (Fast and Discrete Fourier Transforms) to extract in-phase (AM) and quadrature

This work was supported in part by an Intergovernmental Personnel Act, sponsored by U. S. Department of Defense, corresponding author D. A. Howe.

First Author is with the National Institute of Standards and Technology, Boulder, CO 80305 USA (e-mail: David.Howe@nist.gov).
(PM) levels to compute power in $1 \mathrm{~Hz}$ bins. The ADEV related statistics are different in that they extract distributions of frequency-noise in $1 / 2$-octave constant-Q filter bins centered at $\mathrm{f}=1 /(2 \tau)$ as shown in Fig. 1 [4]. Powers-of-two values of AVAR at $\tau=\tau_{0}, 2 \tau_{0}, 4 \tau_{0}, 8 \tau_{0}, 16 \tau_{0}$, etc., distinguish clock power-law noise models [5].

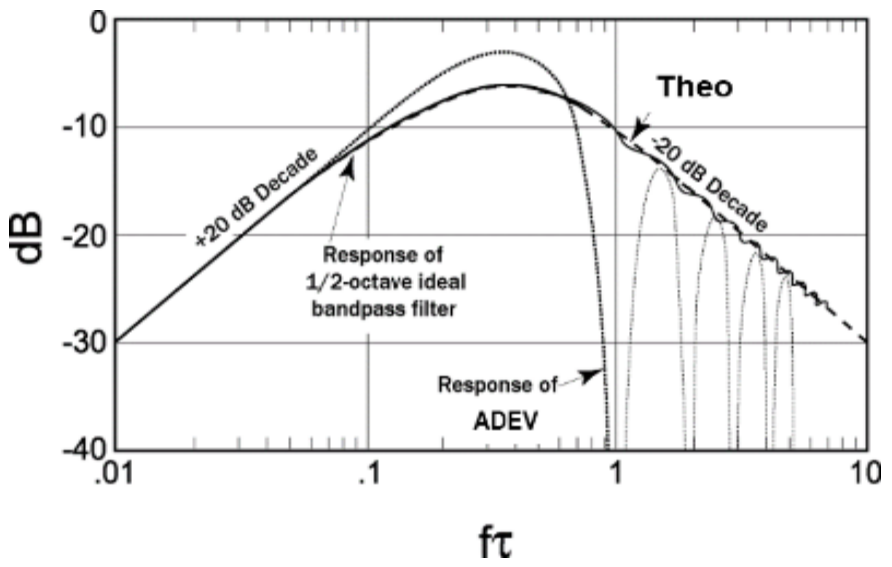

Fig. 1. The digital frequency filter of Allan variance (AVAR), hence, its square-root ADEV. Also shown is Theo1, a statistic created to eliminate the nulls of standard ADEV and match the ideal bandpass response of two cascaded filters, a single-pole high-pass followed by a low-pass with identical break points at $R C=\tau / 2$ (dashed line).

The Allan variance in $2^{j}$ (powers-of-two) increments, $j=$ $0,1,2,3, \ldots$ is given by:

$\operatorname{AVAR}\left(2^{j}\right) \equiv \frac{1}{2^{J-j+1}} \sum_{k=2}^{2^{J-j}}\left(\bar{y}_{2^{j} k_{k}}\left(2^{j}\right)-\bar{y}_{2^{j}(k-1)}\left(2^{j}\right)\right)^{2}$,

where $\bar{y}_{n}\left(2^{j}\right)$ is the average of the last $2^{j}$ points at location $n$ and

$$
\bar{y}_{n}\left(2^{j}\right) \equiv \frac{1}{2^{j}} \sum_{l=0}^{2^{j}-1} y_{n-l} .
$$

From Eq. (1) we see that if there are any data gaps, then the number of equally spaced $1^{\text {st }}$ differences in the summand for each $2^{j}$ level becomes either zero or not representative of the live data. If the raw data are simply joined through gaps, this causes substantial errors and ambiguous results [6]. Thus, imputed data in spaces of dead time is routinely but wrongly by interpolation based on time-domain regression coefficients or frequency-domain models of data before and after a gap. They include Kalman, maximum-likelihood estimation, ARIMA, and min-max entropy analysis [7]. The problems with these

Second Author is with the University of Colorado, Boulder, CO 80303 USA (e-mail: Noah.Schlossber@colorado.edu).

Work of the US Government, not subject to copyright. 
methods are (1) each can become unstable, hence, unreliable, for creating any data for large gaps beyond about $10 \%$ of the live-time data [8], and (2) interpolations do not capture noise properties of live data. The above methods become generally ineffectual at accurately producing ADEV with $10 \%$ or more dead time. In summary, the absence of stochastic noise in gaps (1) causes instability in the above methods as dead time grows, leading to erratic, divergent results, (2) does not represent the live noise, and (3) causes ADEV bias and errors.

$\operatorname{AVAR}(\tau)$ treats 1st-differences of $\bar{y}_{n}$ summand terms in Eq. (1) as ergodic [9]. The squared-difference terms of $\bar{y}_{n}$ in Eq. (1) are simply averaged, so are stationary ergodic for non-convergent red $1 / f$ and $1 / f^{2}$ spectral noises. The $1 / 2$-octave bandpass filter caused by the 1st-diffencing operation itself makes levels separated by octaves in Eq. 1 add up to twice the continuous overall time-average standard variance (SVAR) [5]. In conclusion, $\operatorname{AVAR}(\tau)$ is, in essence, a digital power-spectrum analyzer whose response is from a filter with a $1 / 2$-octave distribution of signal components that occur around center $-f=$ $1 /(2 \tau)$ as shown in Fig. 1 [4]. This property together with ergodicity means that discrete-continuous signals are regarded as infinitely repeating. The FFT and DFT by way of common examples treat a $T$-length block of data as infinitely extended, or circular [3]. The imputation strategy in this writing is based on circular convolution.

$\operatorname{AVAR}(\tau)$ is the mean-square of all sets of a second $\tau$-average frequency subtracted from a prior $\tau$-average frequency. Subtraction is multiplication of a one-period \pm 1 square-wave with a $2 \tau$-length interval within the time-series, called its sampling function, that's squared and averaged. We reason that by a conservation principle that the variance cannot diminish for intervals $2 \tau>T$, justifying circularization of the data [10].

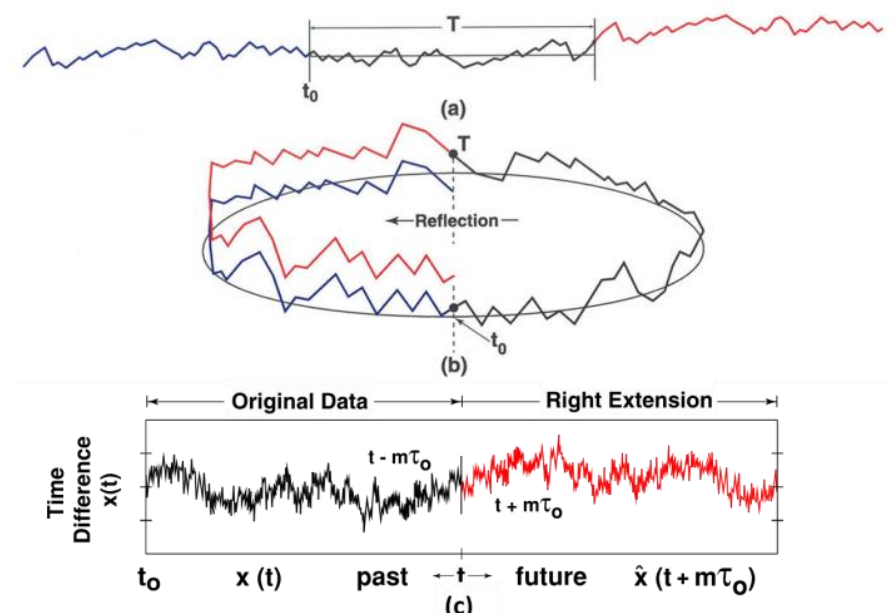

(c)

Fig. 2. (a) and (b): Illustration of circularization of data. (c) Right extension of original data by reflection and inversion. Gaps (dead-times) can be on either side of the original "livetime" data shown between as $t_{0}$ to $t_{0}+T$. Gaps are filled in intervals $t_{0}-T$ to $t_{0}$ to the left of live data and $t_{0}+T$ to $t_{0}+2 T$ to the right. New imputed terms in gaps are iid, that is, independent and identically distributed, as the live data and inherently correlated (self-similar) to the degree of the live data.
Circularization, where the next point after the last point in the time series is the first point, is convolution in the frequencydomain. We remove endpoint discontinuities by reflecting the data run (creating a mirror image) at its last point since AVAR responds identically to data either backward or forward. The Total variance (TOTVAR) is AVAR with this same data extension [11]. Theo1 estimates ADEV by applying multiple sampling functions that are the equivalent of circularizing the data for each $\tau$-value without actually circularizing the data for each $\tau$-value. Theo 1 is added to ADEV calculations at $\tau$-values beyond ADEV's longest-possible $\tau$-value at $T / 2$ to create a combination plot called THEO-H [4]. Computations are as fast as standard ADEV [12]. ADEV fundamentally measures "selfsimilarity" or underlying long-term autocorrelations. Whitenoise FM is uncorrelated, whereas random-walk is fully correlated since it is an integral of white noise. Flicker-noise data is correlated by virtue of being a fractional integral [13]. The gap-filling algorithm described next characterizes mixed noises [14]. This is important for today's atomic-clock comparisons by satellite that are costly and often have gaps, making estimates of a single-clock's ADEV very difficult [15].

\section{STRATEGY FOR FILLING IN DATA GAPS}

A method of data imputation that avoids pitfalls and is effective for up to $100 \%$ dead time is to reflect live data around its end and introduce a phase-slope to match the resumption of live data. Fig. 2(a,b) illustrates circularized live data. With some care, a dataset with gaps filled by imputing extended data preserves the noise characteristic of the live dataset. The original inherent degree of short- and long-term behavior of a time series when measurements have gaps works best by using inverted-reflected extensions for a "right extension" as shown in Fig. 2(c). Simulations of noises are in the next section.

Some might object to the method here because the reflected data is seemingly not iid (independent identically distributed) because, while it is identically distributed, it is not clear that reversed sequences are independent. Independence is determined by how the correlation between any two values of the time series changes as their separation, or "lag-nto", increases [16]. $\operatorname{ADEV}(\tau)$ reports a two-point correlation for lag$\tau$, and $\tau$ cannot exceed $T / 2$ set by the definition of $\operatorname{ADEV}(\tau)$. Processes correlate or decorrelate in proportion to $\tau=2 \tau_{0}, 4 \tau_{0}$, $8 \tau o, 16 \tau$, etc., and we find this behavior to continue into gaps when circularized-reflected data is imputed in gaps [14].

An additional argument is by the equivalent degrees of freedom (EDF), i.e., the number of independent values in a statistical average. EDF is chiefly used to calculate the confidence of an average. We find that EDF always increases with extensions relative to un-extended $\operatorname{ADEV}(\tau)$ calculations, and most dramatically increases for white noise by up to $6 \mathrm{X}$, while less so for flicker and random-walk noise [4].

For a given gap in a dataset of size $n$ missing points, our algorithm is as follows: We take the previous $n$ measurements before the gap (or the following $n$ measurements if there is not enough data before the gap). We then take these $n$ points, 
reverse the temporal indices, and insert them into the gap. Finally, we add a linear slope to this dataset to match the last point of the inserted data matches up with the point of the data White Phase Noise

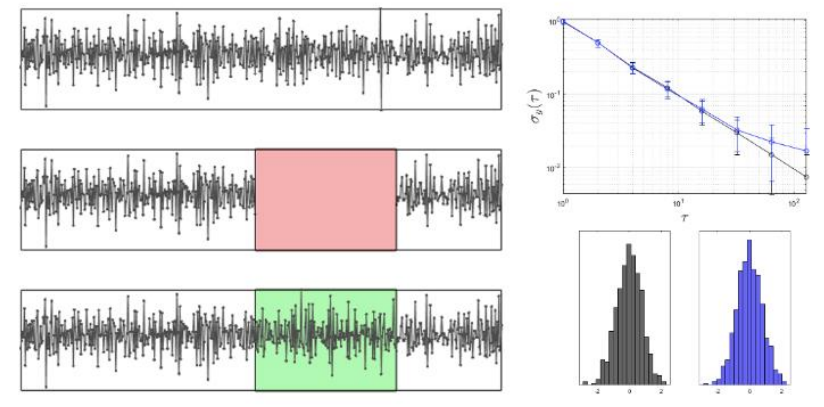

White Frequency Noise
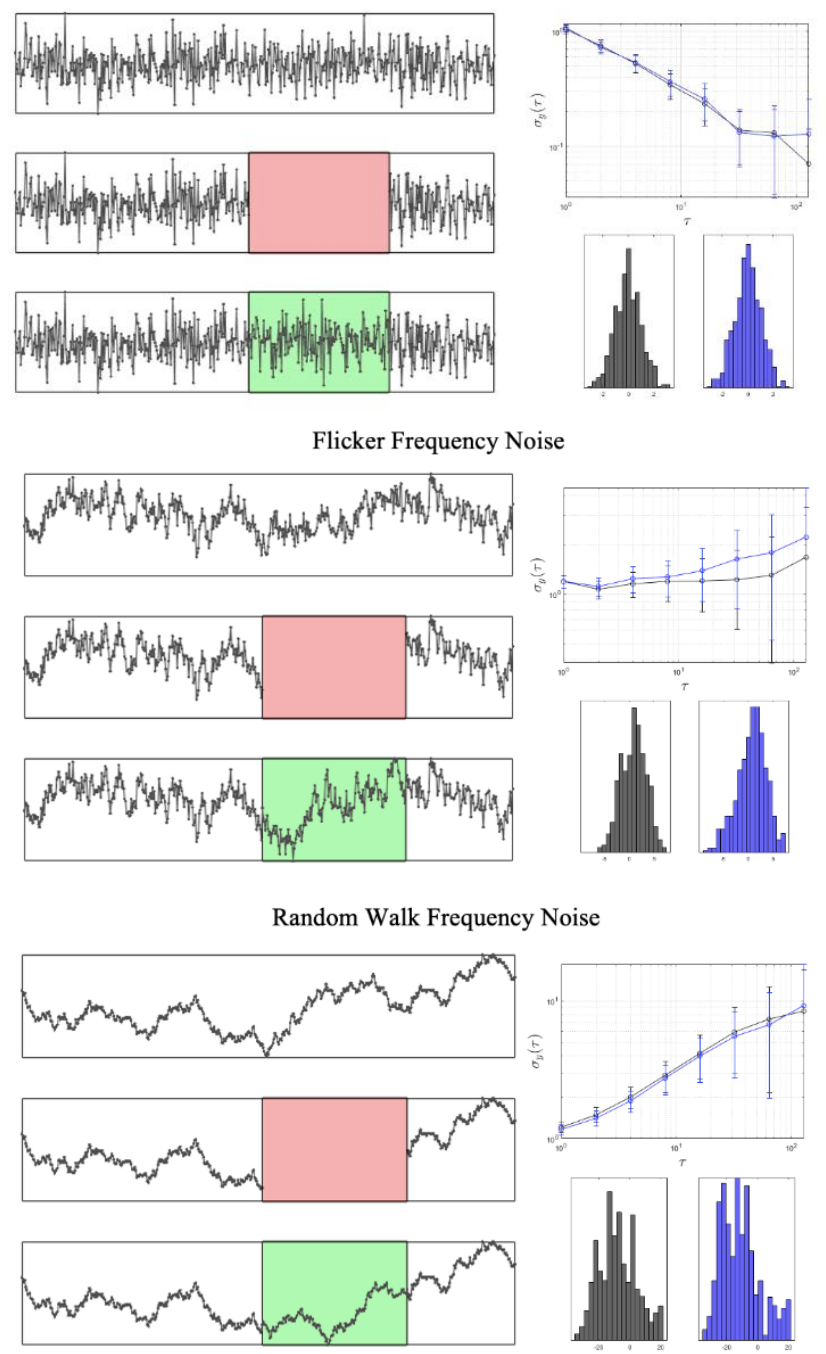

Fig. 3. Application of data imputation algorithm on simulated data of the four predominant noise types. Left: The data reconstruction algorithm. On the top left is the original data, in the middle left the dataset with values removed, and on the bottom left the dataset with imputed values. Top Right: The Allan deviations for the original (black) and recovered (blue) datasets. Bottom Right: The distributions for the original (black in the left box) and recovered (blue in the right box) datasets. after the gap. A naïve single point endpoint-matching can produce an artificial sawtooth pattern in the presence of white PM noise, so a more nuanced approach is used and detailed in the appendix.

For datasets with multiple gaps of dead time, the algorithm that works well is as follows. Find the largest continuous run of data in the set, then impute the gap to its right. Continue to impute consecutive right gaps until the end of the dataset is reached. Then spatially reverse the dataset and continue this algorithm until you reach the beginning of the dataset. In this manner, one can fill all gaps in the dataset provided that the maximum gap size is sufficiently small compared to the initial run of data. In situations where this is not the case, we can impute from the left and right sides and match averages in the middle [14]. If there is a systematic periodic fluctuation imbedded in the noise, such as a diurnal, it may be necessary to move the point of reflection to match the phase of the periodic.

\section{Simulations}

To demonstrate the effectiveness of this algorithm, we have implemented right extensions on 512-length datasets with 150 values missing for the range of clock noise types. The results are shown in Fig. 3. The resulting data is similar to the original in all three major criteria: the noise characteristic, the distribution, and the ADEV curve. Of special importance is that all ADEV measurements on the imputed set lie within error bars for $90 \%$ confidence of the original dataset. An imputation algorithm that fills gaps and is written in Python is in Ref. [14].

\section{CONCLUSION}

Using interpolation to fill intervals of missing measurements (gaps or dead-times) fails to provide acceptable ADEV estimates. In this paper, we impute a "right extension" of live data that is inverted and reversed in sequence to decorrelate it. The Allan family of frequency stability using this imputation preserves the noise distribution and power-law characteristics of the live dataset. The approach proved successful in simulation trials of ADEV using white phase modulation (PM) and frequency modulation (FM), flicker FM, and random walk FM in which a $30 \%$ segment was removed. ADEV agreed with the original data within $90 \%$ confidence limits.

\section{APPENDIX}

ENDPOINT-MATCHING PROCEDURE

FM noises originate from a clock's frequency-determining element, or fundamental resonator. PM noise is wideband jitter from later stages of analog and digital components. To demonstrate why adding a linear slope that simply matches the

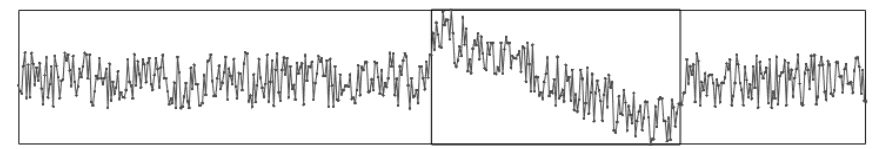

Fig. 4. With simple endpoint matching, the reflect+invert extension on a sample of white noise can result in a visibly incorrect slope associated with imputated data into a gap that's shown as the boxed segment. 
endpoints of the imputed and original data does not work, consider the case of white noise. If the endpoints used to match differ from the local mean, the result is an artificial "sawtooth" feature in the data, as demonstrated in Fig. 4.

Instead, an approach that is suitable for PM and FM noises is to match endpoints based on a filtered version of the data that ignores higher frequency fluctuations. The filter takes the form of a decaying exponential scaled to the length of the dataset $x$ in the frequency domain as:

$$
x_{\text {filtered }}=\operatorname{ifft}\left(\mathrm{ftt}(x) \cdot e^{-2^{3 \frac{|f|}{T}} \tau_{0}^{2}}\right),
$$

where "fft" denotes fast Fourier transform and "ifft" denotes the inverse fast Fourier transform, $T$ is the total length of the imputed data, $\tau_{0}$ is the difference between points in the time series, and $f$ is the Fourier-frequency variable in operator $\mathrm{ftt}(x)$. A qualitative idea of what this filter does can be inferred from its plot in Fig. 5.

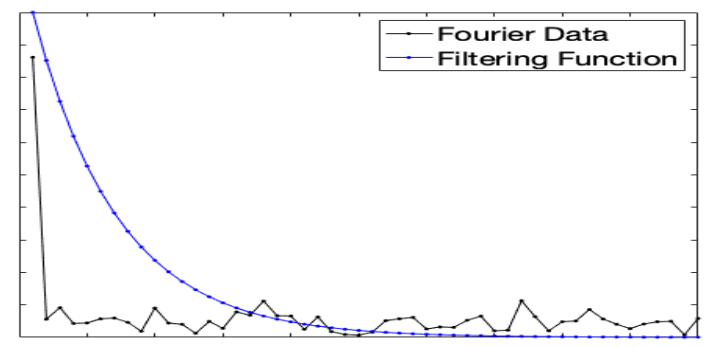

Fig. 5. Plot of the filtering function in the frequency domain, alongside an example fourier spectrum of white noise.

A remaining issue is that the $\mathrm{fft}$ in eq. (3) treats the data as periodic, so when filtered, this will create periodic slopes with steps at the ends that join the first with the last point of the data. The way to avoid these steps is to pad the data with reflected, i.e., flipped, copies of itself on either end. The result is shown in Fig. 6. Most importantly, this technique correctly matches endpoints for not only white PM noise but also all FM noises in Fig. 3

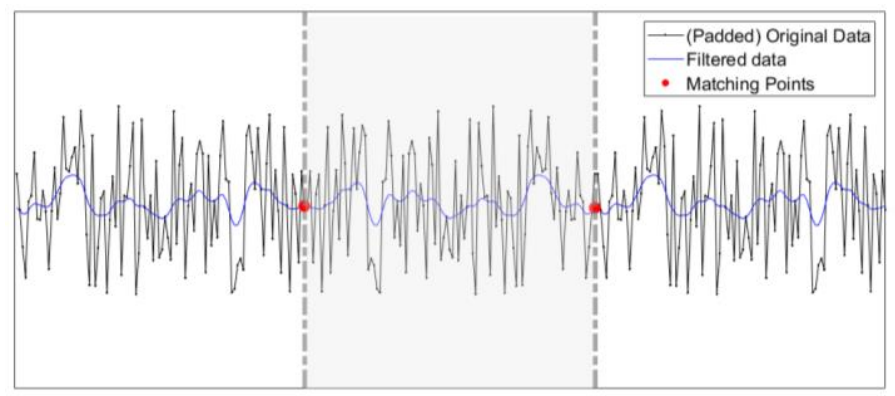

Fig. 6. Plot of the filtering function acting on the data. The middle section is the data segment, padded on either side of the grey dashed line with its own reflection. The thin blue line is then the filtered data with the two filtered are used for endpoint matching. Filtered points are highlighted by the dots in red.
The algorithm is then as follows:

Use the filter on the block of data to the right and to the left of the gap. Then take the imputation of the filtered dataset and add a linear slope to the unfiltered imputed data such that its difference of endpoints is equal to the difference of the filtered endpoints.

\section{REFERENCES}

[1] P1139TM/D1 (Draft) Standard Definitions of Physical Quantities for Fundamental Frequency and Time Metrology - Random Instabilities, IEEE-SA Standards Board, The Institute of Electrical and Electronics Engineers, New York 10016-5997, USA (in process). Also, Int'l Telecom Union Recommendation ITU-R TF.538-5 - Measures for random instabilities in frequency and time (phase).

[2] D.B Sullivan, D.W Allan, D.A Howe, and F.L Walls, eds., "Characterization of clocks and oscillators," Natl. Inst. Stand. Technol. Technical Note 1337, http://tf.nist.gov/timefreq/general/pdf/868.pdf (March 1990).

[3] Howe, D.A., Allan, D.W., Barnes, J.A., "Properties of Signal Sources and Measurement Methods," Proc IEEE International Symp on Freq Control, May, 1981, pp. A1-A47.

[4] D. A. Howe, "TheoH: a hybrid, high-confidence, statistic that improves on the Allan deviation," Metrologia, 43 (2006) S322-S331.

[5] N. Schlossberger and D. Howe, "Analysis of powers-of-two calculations of the Allan variance and their relation to the standard variance," Proc. 2019 Joint Mtg. IEEE Intl. Freq. Cont. Symp. and EFTF Conf., 5 p.

[6] P. Lesage, "Characterization of Frequency Stability: Bias Due to the Juxtaposition of Time-Interval Measurements," IEEE Trans. Instrum. Meas., Vol. IM-32, No. 1, pp.204-207, March 1983.

[7] NIST/SEMATECH e-Handbook of Statistical Methods, http://www.itl.nist.gov/div898/handbook/ The difficulty of gap filling is discussed along with proposed strategies for certain kinds of data in: D. Mondal and D. B. Percival, "Wavelet Variance Analysis for Gappy Time Series," Annals of the Institute of Statistical Mathematics, 62, no. 5, pp. 943-966 (2010).

[8] G. Box and G. Jenkins, Time Series Analysis, forecasting and control, Holden-Day, San Francisco, 1970.

[9] Halmos, P. R. Letures on Ergodic Theory, The Mathematical Society of Japan, Kenkyushu Printing Co., Ltd., Tokyo, 1956.

[10] D. A. Howe, "Circular Representation of Infinitely Extended Sequences," Proc. 1995 IEEE Intl. Freq. Cont. Symp., pp. 337-346.

[11] C.A. Greenhall, D.A. Howe and D.B Percival, "Total Variance, an Estimator of Long-Term Frequency Stability", IEEE Trans. Ultrasonics, Ferroelectrics and Freq. Contrl., Vol. UFFC-46, No. 5, pp. 1183-1191, Sept. 1999.

[12] B. Lewis, "A Fast Algorithm for Calculation of Thêo1", IEEE Transactions on Ultrasonics, Ferroelectrics and Frequency Control. 67, 10, pp. 2187-2190, 1 Oct 2020.

[13] B. Mandelbrot on Statistical Self-Similarity and Fractional Dimension, Science 05 May 1967, Vol. 156, Issue 3775, pp. 636-638.

[14] D. A. Howe, C. Champagne, and N. Schlossberger, "Time Series Imputation Algorithm," IEEE Signal Processing Letters, in process. This paper includes the algorithm described herein.

[15] D. Yu, M. A. Weiss, and T. E. Parker, "Uncertainty of a frequency comparison with distributed dead time and measurement interval offset," Metrologia 44, pp 91-96.

[16] Beran, J., Statistics for Data with Long-range Dependence, Statistical Science, 7, pp. 404-427 (1996). 